\title{
Method of control of machining accuracy of low-rigidity elastic-deformable shafts
}

\section{Abstract}

The paper presents an analysis of the possibility of increasing the accuracy and stability of machining of low-rigidity shafts while ensuring high efficiency and economy of their machining. An effective way of improving the accuracy of machining of shafts is increasing their rigidity as a result of oriented change of the elasticdeformable state through the application of a tensile force which, combined with the machining force, forms longitudinal-lateral strains. The paper also presents mathematical models describing the changes of the elastic-deformable state resulting from the application of the tensile force. It presents the results of experimental studies on the deformation of elastic low-rigidity shafts, performed on a special test stand developed on the basis of a lathe. An estimation was made of the effectiveness of the method of control of the elastic-deformable state with the use, as the regulating effects, the tensile force and eccentricity. It was demonstrated that controlling the two parameters: tensile force and eccentricity, one can improve the accuracy of machining, and thus achieve a theoretically assumed level of accuracy.

\section{Keywords}

low rigidity shaft, control of machining accuracy, mathematical models of machining, efficiency, mechanics of machine tools

\author{
Antoni Świć ${ }^{1}$, Dariusz Wołos ${ }^{1}$, \\ Grzegorz Litak ${ }^{*}, 2$ \\ ${ }^{1}$ Institut of Technological Information Sys- \\ tems, Lublin University of Technology, \\ Nadbystrzycka 36, PL-20-618 Lublin, Poland \\ ${ }^{2}$ Department of Applied Mechanics, Lublin \\ University of Technology, \\ Nadbystrzycka 36, PL-20-618 Lublin, Poland \\ Received 06 Feb 2013 \\ In revised form 03 May 2013 \\ *Author email: g.litak@pollub.pl
}

\section{INTRODUCTION}

A half or all machine parts are rotating elements: shafts (ca. 40\%), discs, sleeves, cylinders etc. Among those, up to $12 \%$ are low-rigidity shafts (Pus et al., 1982).

A highly important, and at the same time complex problem is the achievement of the assumed accuracy of machining and operational reliability of low-rigidity shafts. Such shafts are elements of many assemblies of various machines and devices, and find applications in, among others, aerospace industry, precision mechanics, tool-making industry (special tools), automotive industry. They are characterised by disproportion in overall dimensions and low rigidity in specific sections and directions. Stringent requirements are also applied in terms of the geometric shape, mutual positioning of surfaces, linear dimensions and quality of surface finish. 
The specific nature of machining of similar parts causes that the primary difficulty relates to the achievement of the required parameters of the accuracy of form, dimensions and surface quality. Low inherent rigidity and relatively low rigidity of the shaft, compared to the stiff assemblies of the machine tool, cause the appearance of vibrations under specific conditions. The process of machining interferes with and destabilises many factors (large free distortions of shafts, vibrations in the tool-object system, breaking of chips etc.), which causes a reduction in the accuracy of machining (Cardi et al., 2008; Hassuiand and Diniz, 2003; Jianliang and Rongdi 2006; Litak et al., 2004; Litak and Rusinek 2012; Qiang, 2000; Altintas, 2000).

The traditional methods of achieving accurate machining of low-rigidity shafts, based on multi-pass machining, lowered parameters of machining, steadies and additional treatments and manual lapping, cause a significant lowering of efficiency, and in many cases preclude the achievement of required reliability; also, they are incompatible with the contemporary requirements of automation, they are uneconomical and inefficient.

The initial studies on the dynamic response of a rotation shaft subjected to a moving load has been done by Katz et al. (1988). Especially, they focused on the dynamical effects accounted by different approaches: Euler-Bernoulli, Rayleigh, and Timoshenko beam models for a simply supported rotating shaft leading to the changes in rotor response. This problem was generalized to a threedirectional load moving in the axial direction by Ouyang and Wang (2012).

In the context of a regenerative cutting process, chatter vibrations response of the workpiece modelled as a flexible beam were also studied by Altintas (2000), Tusty (2000), Chen and Tsao (2006), and more recently by Bisu et al. (2009a; 2009b), Cahuc et al. (2010), Han et al. (2012).

\section{MATHEMATICAL MODELS OF MACHINING OF ELASTIC-DEFORMABLE SHAFTS}

One of the effective ways of improving the accuracy of machining of parts of this type is increasing their rigidity as a result of oriented change of their elastic-deformable state, through the application of a tensile force which, combined with the machining force, forms longitudinal-lateral strains. As shown by experimental studies, increase of rigidity of parts with diameters from 2 to $6 \mathrm{~mm}$ and length from 100 to $300 \mathrm{~mm}$, with their loading with a tensile force within the range from 980 to $1960 \mathrm{~N}$, leads to a reduction of elastic strain by from 80 to $20 \%$, respectively. Furthermore at diameters $d=8-12 \mathrm{~mm}$ such a loading reduce elastic stain by $5-7 \%$. Increasing of $d>16 \mathrm{~mm}$ at a given length has practically no effect on the value of static stiffness and, correspondingly, on the deformation of the parts (Jianliang and Rongdi, 2006; Swić et al. 2010).

Analysis of the effect of the tensile force on the static rigidity of machined elements can be performed with the use of the model in Table 1, line 1. The model does not provide an adequate description of the behaviour of the elastic line at various methods of fixing. This means that, in the model in question, the element fixed in the tailstock of the lathe has both the possibility of linear displacement along the axis of the part and the possibility of free rotation of the section at the point of fixing. In many cases, such a method of fixing does not lead to a reduction of deformation in the machining zone.

With the application of the tensile force, the fixing of a shaft can be realized by means of a spring sleeve. Such a method of fixing can be interpreted as rigid fixing, with the possibility of axial play (Tab. 1, lines 2 and 3). 
To minimise elastic deformation, it is also possible to control the angle of rotation of the part section at the point of fixing, through the application of a tensile force shifted with relation of the axis of the centres (Świć et al. 2010). This kind of fixing can also be represented as a moving rotary support (Tab. 1, line 4).

The fundamental feature of the presented schematic is the application of the control moment at the point of fixing of the machined part - through eccentric tension. The application of a single controllable force factor - eccentric tension - permits the generation of two force factors at any predefined section of the part, and in the machining zone in particular: the longitudinal force $F_{x 1}$ and the bending moment $M_{2}=F_{x 1} \cdot e$, counteracting the machining forces, i.e. the oriented elasticdeformable state of the shaft.

The application of tensile forces with a shift relative to the axis of the centres at both ends of the machines shaft - in this case the fixing can be represented as a moving rotary support (Tab. 1, line 5) - permits the control of the position of the part axis from two sides at any position of the cutting tool, relative to the length of machining. Moreover, it is possible to use a special fixture for mobile tensioning in the machining of long shafts with low rigidity (Tab. 1, line 6).

The specifics of elastic-deformable loading of low-rigidity parts, with eccentric compression in machining operations, are taken into account in model 7 (Tab. 1).

In Table 1 the following symbols are used:

$\begin{array}{ll}F_{x 1} & \text { tensile force; } \\ F_{z g} & \text { bending force; } \\ F_{c}, F_{p}, F_{f} & \text { are two bending force and axial components; } \\ e & \text { eccentricity of tensile force in tension; } \\ M_{1} & \text { moment generated by the axial component } F_{f} \text { of the machining force; } \\ x_{1}, x_{2}, x_{3} & \text { current coordinates at each of the sections; } \\ a & \text { distance between the cutting edge (point of load application) and the point } \\ & \text { of fixing of the part in the spindle; } \\ & \text { other characteristic distances along the shaft; } \\ d & \text { diameter of the machined part; } \\ L & \text { length of the shaft; } \\ Q_{0} \text { and } M_{0} & \text { initial parameters: perpendicular force and moment at the point of the fixing } \\ & \text { of the part, respectively. }\end{array}$

One of the methods that permit the generation of a mathematical model describing the kind of elastic line, with relation to the part parameters and to the parameters of the process of machining (loading forces) is the energy method of Ritz, by means of which the deformation functions were obtained for a tensioned rod with an end fixed rigidly (Tab. 1, line 2).

Another method permitting the achievement of results with practical utility is the generation of a description of the elastic line of a low-rigidity part in lateral-longitudinal bending, in the form of a system of differential equations of the fourth order, with a constant coefficient. With the occurrence of concentrated forces and moments dividing the shaft into sections the following differential equations can be written (axial tension - model 3) at each of the sections:

Latin American Journal of Solids and Structures 11(2014) $260-278$ 


$$
y_{i}^{I V}-\alpha^{2} y_{i}^{\prime \prime}=0
$$

where: $\alpha=\sqrt{\frac{F_{x 1}}{E I}}, E$ - modulus of elasticity, $I$ - moment of inertia of the section.

In the simplest case, the only disturbance of the elastic line is located at the point of machining, i.e.

$$
i=\in\{1,2\}
$$

The solution of equation (1) can be written in the simplified single mode form (for symmetric situation - model 2 instead of hyperbolic functions the trigonometric ones are used) (Young et al., 2003):

$$
y_{i}\left(x_{i}\right)=A_{i} \sinh \alpha x_{i}+B_{i} \cosh \alpha x_{i}+C_{i} x_{i}+D_{i}
$$

and in the case of (2):

$$
\left\{\begin{array}{l}
y_{1}\left(x_{1}\right)=A_{1} \sinh \alpha x_{1}+B_{1} \cosh \alpha x_{1}+C_{1} x_{1}+D_{1} \\
y_{2}\left(x_{2}\right)=A_{2} \sinh \alpha x_{2}+B_{2} \cosh \alpha x_{2}+C_{2} x_{2}+D_{2} .
\end{array}\right.
$$

Taking into account that $M_{1}\left(x_{1}\right)=E I \cdot y_{1}^{\prime \prime} ; \quad F_{1}\left(x_{1}\right)=E I \cdot y_{1}^{\prime \prime \prime} ; \quad M_{2}\left(x_{2}\right)=E I \cdot y_{2}^{\prime \prime} ;$ $F_{2}\left(x_{2}\right)=E I \cdot y_{2}^{\prime \prime \prime}$, from the boundary conditions (column 3, Tab. 1) in each system of coordinates at $x_{1}=0$, deformation $y_{1}(0)=0$, angle of rotation of section $y_{1}^{\prime}(0)=0$ and $E I \cdot y_{1}^{\prime \prime}(0)=M_{0}$, $E I \cdot y_{1}^{\prime \prime \prime}(0)=Q_{0}$, the constant coefficients were determined as follows:

$$
A_{1}=\frac{Q_{0}}{E I \alpha^{2}}, B_{1}=\frac{M_{0}}{E I \alpha^{2}}, C_{1}=-\frac{Q_{0}}{E I \alpha^{2}}, D_{1}=-\frac{M_{0}}{E I \alpha^{2}},
$$

and the equation of deformations on section I, taking into account (5) and the terms for the determination of $\alpha$ can be written as:

$$
y_{1}\left(x_{1}\right)=\frac{Q_{0}}{\alpha F_{x 1}}\left(\sinh \alpha x_{1}-\alpha x_{1}\right)+\frac{M_{0}}{F_{x 1}}\left(\cosh \alpha x_{1}-1\right)
$$

Coefficients $A_{2}, B_{2}, C_{2}, D_{2}$ were expressed from the boundary conditions $x_{2}=0$, conditions of balance and compensation of deformations $y_{1}^{\prime}(a)=y_{2}^{\prime}(0), \quad E I y_{1}^{\prime \prime}(a)=E I y_{2}^{\prime \prime}(0)$, $E I y_{1}^{\prime \prime}(a)+F_{z g}=E I y_{2}^{\prime \prime \prime}(0)=0$ and they are equal to: 


$$
\begin{gathered}
A_{2}=\frac{Q_{0} \cosh \alpha a+M_{0} \alpha \sinh \alpha a+F_{z g}}{\alpha^{3} E I}, \\
B_{2}=\frac{Q_{0} \sinh \alpha a+M_{0} \alpha \sinh \alpha a}{\alpha^{3} E I}, \\
C_{2}=\frac{Q_{0} \cosh \alpha a+M_{0} \alpha \sinh \alpha a+F_{z g}}{\alpha^{3} E I}, \\
D_{2}=\frac{Q_{0} \sinh \alpha a+M_{0} \alpha \sinh \alpha a}{\alpha^{3} E I},
\end{gathered}
$$

And the equation of deformation on section II can be written as:

$$
y_{2}\left(x_{2}\right)=\frac{Q_{0} \operatorname{ch} \alpha a+M_{0} \alpha \operatorname{sh} \alpha a+F_{z g}}{\alpha F_{x 1}}\left(\sinh \alpha x_{2}-\alpha x_{2}\right)+\frac{Q_{0} \sinh \alpha a+M_{0} \alpha \cosh \alpha a}{\alpha F_{x 1}}\left(\cosh \alpha x_{2}-1\right) .
$$

Equations of deformations on sections I and II, for the case of model 4 (Tab. 1) and the other cases of loading considered, obtained in an analogous manner, are presented in column 4, Tab. 1.

The values of the initial parameters $Q_{0}$ and $M_{0}$ were determined at extreme conditions at the end of the rod:

$$
y_{2}^{\prime}(L-a)=0
$$

and the equations of deformations at the end of the deformed rod:

$$
y_{1}(a)=-y_{2}(L-a)
$$

The results of solving equations (9) and (10) are given in column 5, Tab. 1.

At eccentric tension (model $4, F_{f} \neq 0, e \neq 0$ ) the differential equations (1) on each of sections I and II have the form:

$$
\begin{aligned}
& y_{1}^{I V}-\alpha_{1}^{2} y_{1}^{\prime \prime}=0 \\
& y_{2}^{I V}-\alpha_{2}^{2} y_{2}^{\prime \prime}=0
\end{aligned}
$$

where: $\alpha_{1}=\sqrt{\frac{F_{x 1}-F_{f}}{E I}}, \alpha_{2}=\sqrt{\frac{F_{x 1}}{E I}}$, and the solution of (11) and (12) is written as (3), taking into account $\alpha_{1}$ on section I. Substituting the boundary conditions, conditions of balance and conditions of simultaneity of deformations (column 3, Tab. 1) into equations (11) and (12), a description of deformations was obtained, as presented in column 4, Tab. 1.

The initial parameters $Q_{0}$ and $M_{0}$ were determined through conditions (9) and the equation of balance: 


$$
\sum M(L)=Q_{0} L+M_{0}+F_{f} \frac{d}{2}+F_{z g}(L-a)-F_{x 1} e=0
$$

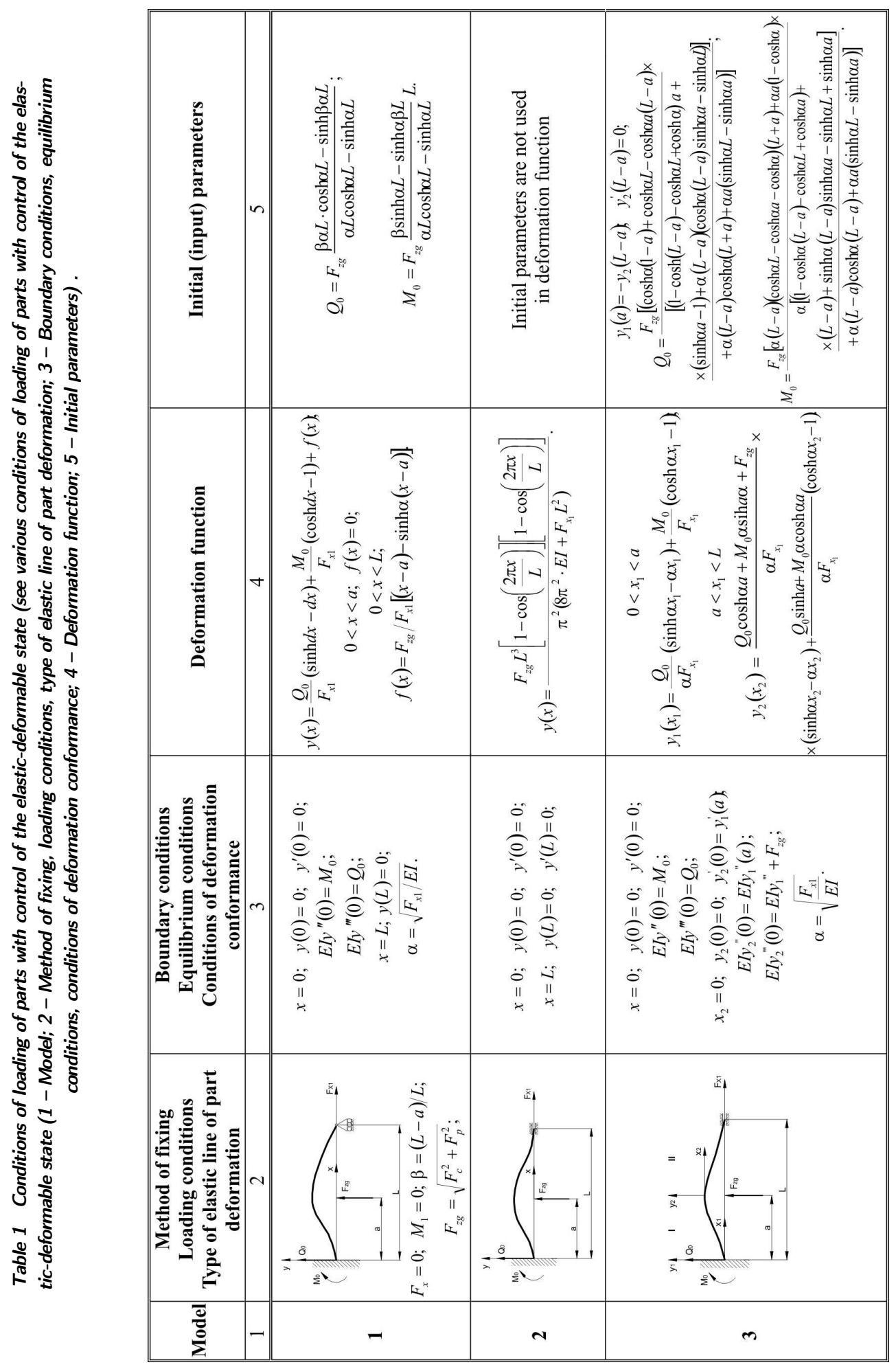

Latin American Journal of Solids and Structures 11(2014) 260 - 278 


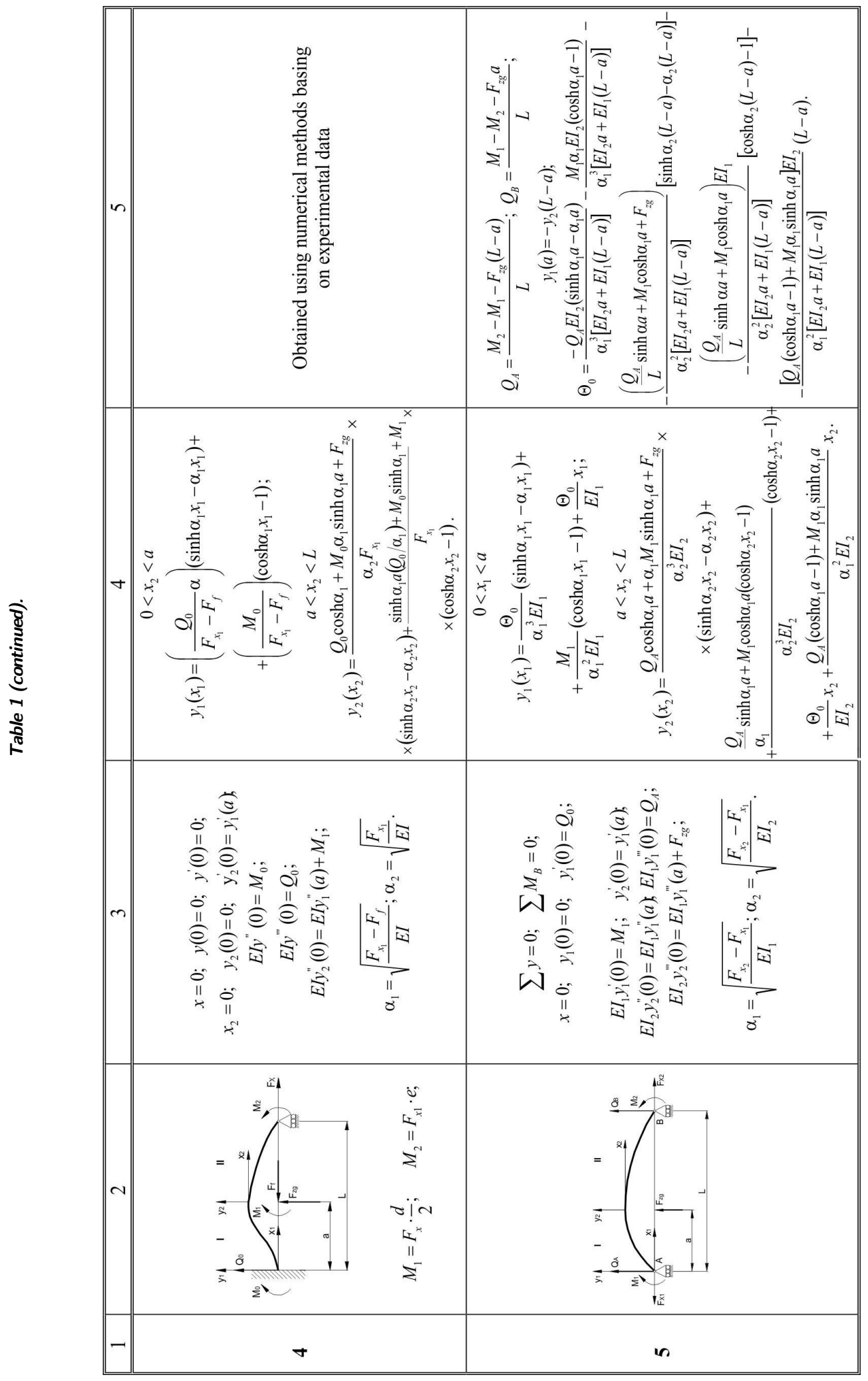

Latin American Journal of Solids and Structures 11(2014) 260 - 278 


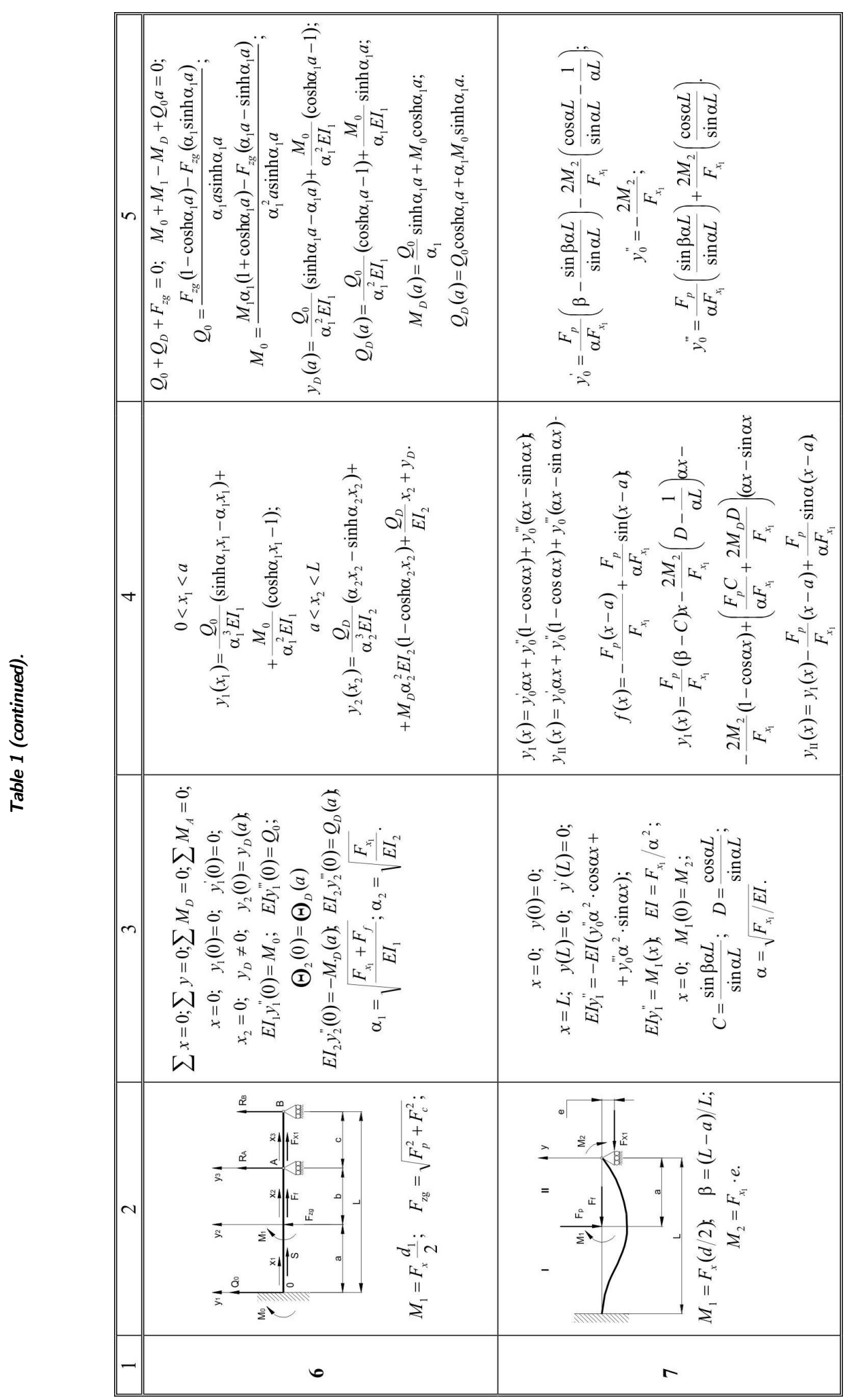

Latin American Journal of Solids and Structures 11(2014) $260-278$ 
and the obtained values of $Q_{0}$ and $M_{0}$ are given in column 5, Tab. 1 .

A specific feature of the problem of control of elastic deformations of an elastic-deformable part under consideration, with two-sided eccentric tension (Tab. 1, line 5), is the ease of determination of the initial parameters $Q_{0}$ at known $M_{1}=F_{x 1} \cdot e_{1}$ and $M_{2}=F_{x 2} \cdot e_{2}$ from the conditions of the balance of forces, relative to the axes of coordinates $\left(\sum Y=0\right)$ and of the balance of moments ( $\sum M_{b}=0-$ columns 3 and 5 ). At the same time, it becomes more complicated to determine the angle of rotation $Q_{0}$, at which the term sought was obtained from equations (10) and presented in column 5.

For a long part loaded with a tensile force, the calculation schematic was presented in the form of a beam twice statically indeterminate (Tab. 1, line 6), loaded with bending force $F_{z g}$, moment $M_{1}=F_{f} \cdot \frac{d}{2}$ and longitudinal forces $F_{f}$ and $F_{x 1}$. The values of the forces and moments are definite, with $F_{x 1}>F_{f}$ and $F_{f}>0$, therefore to the left from point $A$ the beam is always in a state of tension, and the value of $F_{f}$ can be negative as the direction of the working travel $f$ changes. In the case of diameter equal to $d_{1}$ on the left section of the part (from point $D$ to 0 ) and diameter $d_{2}$ (to the right from $D$ ), the axial moments of inertia of the cross-section will equal, respectively,: $I_{1}=\frac{\pi d_{1}^{4}}{64}, I_{2}=\frac{\pi d_{2}^{4}}{64}$, and parameters $\alpha_{2}=\sqrt{\frac{F_{x 1}}{E I_{2}}}, \alpha_{1}=\sqrt{\frac{F_{x 1}+F_{f}}{E I_{1}}}$. When support $A$ moves to the right, point $D$ moves with it at the same velocity, and distance $b$ has a constant value. Dimension $a$ changes within the range of $0 \leq a \leq(1-a)$. To solve the double statically indeterminate problem of longitudinal-lateral bending of the beam, under loading with the moving tensile force $F_{x 1}$, the left and the rights parts of the beam were analysed.

From the boundary conditions and the conditions of mutual interaction of deformations (Tab. 1, line 6) the functions of deformations (column 4) and the initial parameters (column 5) were obtained.

The results of modelling of the values of elastic deformations of the part within the machining zone $(x=a)$ are presented in Fig. 1, the numbers of analytical relations corresponding to the numbers designating the models in Table 1 . Relation 5 was obtained experimentally, with the part fixed in the grip of the spindle and in the spring sleeve of the tailstock, with no possibility of cross-section rotation at the point of fixing (model 3). 

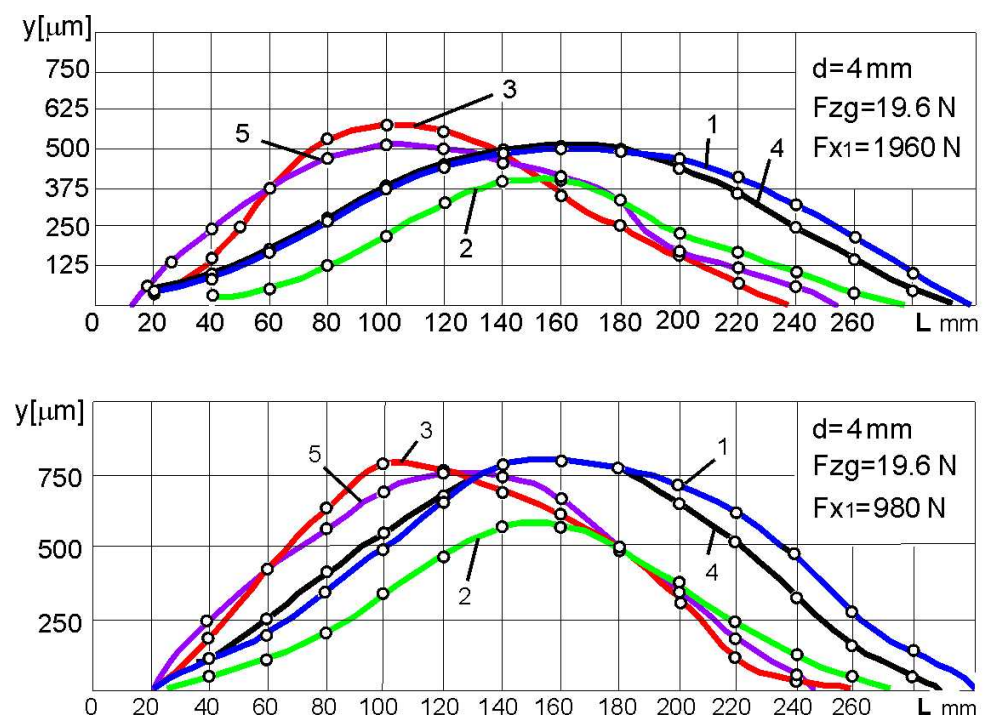

Figure 1 Estimated relations of elastic deformation changes of a shaft (numbers from 1 to 5 correspond to the numbers designating the models in Table 1).

\section{EXPERIMENTAL STUDIES}

The experimental studies of elastic deformations of low-rigidity shafts were conducted on a special test stand, constructed on the basis of a lathe (Fig. 2).

A shaft (1) is aligned in the lathe grip between a standard compression dynamometer (2) type DOSM-3-02 (measurement range from 19.6 to $196.0 \mathrm{~N}$ ), mounted by means of a bracket (3) in the cutter holder (4) of the lathe. The radial component of the cutting force $F_{p}$ was estimated using the dynamometer (2). Registration of elastic deformations was conducted by means of an electromagnetic displacement transducer (9) with a recorder unit (10). The transducer (9) was mounted in a holder (8) on a plate (6) positioned on the guide rails (5 and 7).

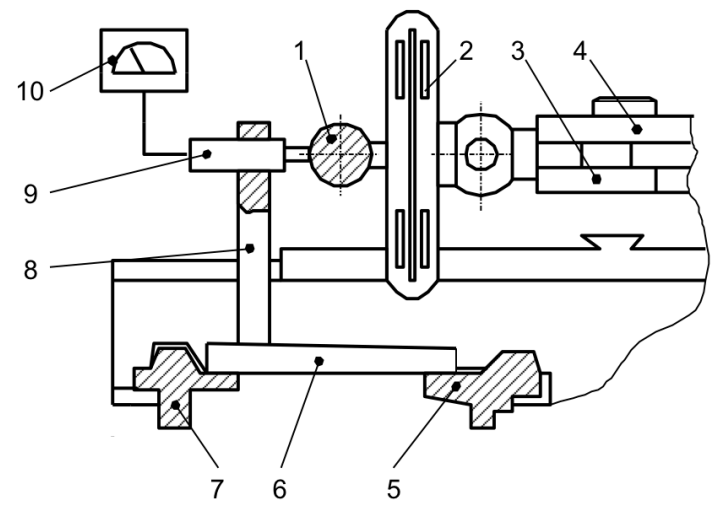

Figure 2 Schematic of the experimental stand for the testing of elastic deformations of parts. 
The experimental stand was used to test the elastic deformations of shafts with diameters $d=2-18 \mathrm{~mm}$ and lengths of 100, 200 and $300 \mathrm{~mm}$. The maximum deformation of the part, under tension with the axial force $F_{x 1}$, decreases in a non-linear manner in accordance with the equations given above (Tab. 1).

Experimental results (model 1) are shown in Fig. 3. The shaft was loaded with axial force $F_{\mathrm{x} 1}=$ $1960 \mathrm{~N}$ (a) and $980 \mathrm{~N}$ (b), respectively, while the lateral force was $F_{\mathrm{zg}}=19.6 \mathrm{~N}$. The measurements accuracies were $0.4 \mathrm{~N}$ and $0.01 \mathrm{~mm}$ for $F_{\mathrm{x} 1}$ axial forces and displacements, respectively.

Based on the further experiments performed (to model 2) one can state that the discrepancy between the analytical results and the experimental data is from 3 to $12 \%$. The calculations were made with the use of model 4 , with the assumption that $F_{x 1} \neq 0, e=0$ fully correspond to the data obtained from model 1.

Certain discrepancies between the results could also result from the assumptions adopted in the selection of calculation scheme.

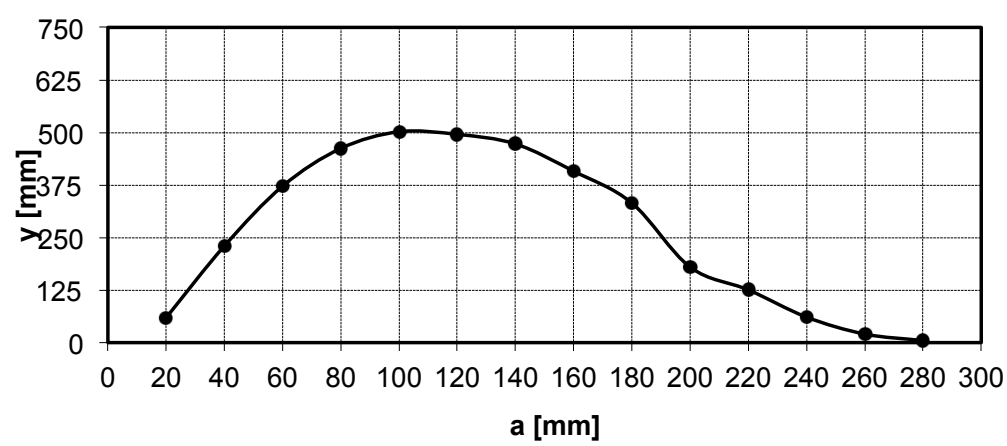

a)

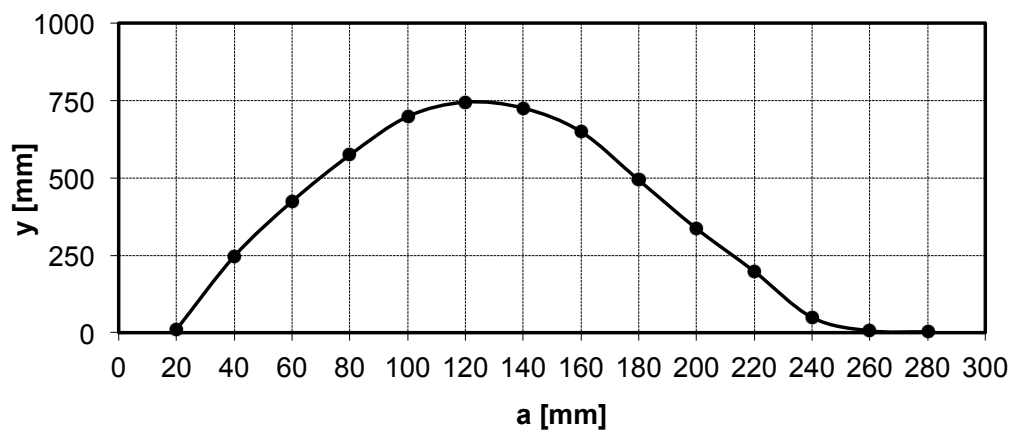

b)

Figure 3 Experimental dependence of elastic shaft deformation (of length $L=300 \mathrm{~mm}$ and diameter $d=4 \mathrm{~mm}$ ) loaded with axial force $F_{\mathrm{x} 1}=1960 \mathrm{~N}(\mathrm{a})$ and $980 \mathrm{~N}(\mathrm{~b})$. The lateral force $F_{\mathrm{zg}}=19.6 \mathrm{~N}$, fitted according to the model shown in Table 1, case 3 .

On the basis of the calculations of elastic deformations caused by axial tension, and also on the basis of experimental data, it can be stated that at any point on the part $(L / d=15-50$ within the range of shaft diameters under consideration), and at the point of machining in particular, situated 
directly beneath the cutting edge $(x=a)$, the value of elastic deformation of the shafts tested can be significantly reduced through the selection of a suitable tensile force $F_{x 1}$ and eccentricity $e$ (Halas et al., 2008; Świć et al. 2011).

In the development of technological methods for the control of grinding accuracy of low-rigidity shafts the elastic-deformable state can be generated by means of longitudinal compressive forces, shifted relative to the axis of the centres or applied additionally to the ends of the parts by means of bending moments.

\section{OPTIMISATION OF PARAMETERS OF AN ELASTIC-DEFORMABLE STATE}

The calculation scheme of the forces acting on the shaft and the elastic line of the shaft are presented in Table 1 (line 7). The application of compressive forces, shifted relative to the axis of the centres, was considered as method of generation of a bending moment applied to the face of a lowrigidity shaft and as a technological premise for the control of machining accuracy.

In the case of a beam compressed with a longitudinal force and transmitting any lateral force, the solution of equation (1) can be presented in the form:

$$
y=y_{0}+y_{0}^{\prime} \alpha x+y_{0}^{\prime \prime}(1-\cos \alpha x)+y_{0}^{\prime \prime \prime}(\alpha x-\sin \alpha x)+f(x),
$$

where: $y_{0}, y_{0}^{\prime}, y_{0}^{\prime \prime}, y_{0}^{\prime \prime \prime}$ - deformation, angle of rotation, second and third derivatives at the origin of the system of coordinates, respectively,; $f(x)$ - function of the effect of lateral loads.

The equations of the lines of elastic deformations on sections I and II have the form of:

$$
\left\{\begin{array}{c}
y_{I}=y_{0}^{\prime} \alpha x+y_{0}^{\prime \prime}(1-\cos \alpha x)+y_{0}^{\prime \prime \prime}(\alpha x-\sin \alpha x), \\
y_{I I}=y_{0}^{\prime} \alpha x+y_{0}^{\prime \prime}(1-\cos \alpha x)+y_{0}^{\prime \prime \prime}(\alpha x-\sin \alpha x)+f(x) .
\end{array}\right.
$$

The initial parameters, in accordance with the conditions adopted earlier (Tab. 1) are as follows:

$$
\begin{gathered}
y_{0}^{\prime}=-\frac{F_{z g}}{\alpha F_{x 1}}\left\{\frac{[\alpha L(\beta-1+\cos \beta \alpha L)-\sin \alpha L](\cos \alpha L)}{\alpha L \cos \alpha L-\sin \alpha L}-(1-\cos \beta \alpha L)\right\}+ \\
+\frac{M_{2}}{F_{x 1}}\left[\frac{(\alpha L \sin \alpha L+\cos \alpha L-1)(1-\cos \alpha L)}{\alpha L \cos \alpha \beta L-\sin \alpha L}+\sin \alpha L\right] \\
y_{0}^{\prime \prime \prime}=\frac{F_{z g}}{\alpha F_{x 1}}\left[\frac{[\alpha L(\beta-1+\cos \beta \alpha L)-\sin \alpha L](\beta \alpha L)}{\alpha L \cos \alpha L-\sin \alpha L}\right]- \\
\frac{M_{2}}{F_{x 1}}\left(\frac{\alpha L \sin \alpha L+\cos \alpha L-1}{\alpha L \cos \alpha L-\sin \alpha L}\right)
\end{gathered}
$$


where: $\beta=\frac{L-a}{L}, F_{x 1}-$ in the given case - compressive force.

After integration and transformation of (15), the equation of the bending moment on section I was obtained:

$$
M_{1}(x)=-\frac{F_{x 1}}{\alpha^{2}}\left(y_{0}^{\prime \prime} \alpha^{2} \cos \alpha x+y_{0}^{\prime \prime \prime} \alpha^{2} \sin \alpha x\right)
$$

Taking into account that at $x=0, M_{1}(0)=M_{2}$ it follows from (18) that $M_{2}=\frac{F_{x 1}}{\alpha^{3}}$ and, correspondingly, $\quad y_{0}^{\prime \prime}=\frac{-M_{2}}{F_{x 1}}$. After considering the function of the effect of lateral loading

$$
f(x)=-\frac{F_{z g}(x-a)}{F_{x 1}}+\frac{F_{z g}}{\alpha F_{x 1}} \sin (x-a),
$$

the equation of deformations on the shaft sections ultimately assumes the form:

$$
\left\{\begin{aligned}
y_{1}(x)=- & \frac{F_{z g}}{F_{x 1}}[A(1-\cos \varepsilon L)-(1-\cos \beta \alpha L)] x+\frac{M_{2}}{F_{x 1}}[B(1-\cos \alpha L+\sin \alpha L)] \alpha x- \\
& \frac{M_{2}}{F_{x 1}}(1-\cos \alpha x)+\left(\frac{F_{z g} A}{\alpha F_{x 1}}-\frac{M_{2} B}{F_{x 1}}\right)(\alpha x-\sin \alpha x), \\
y_{I I}(x)= & y_{I}(x)-\frac{F_{z g}}{F_{x 1}}(x-a)-\frac{F_{z g}}{\alpha F_{x 1}} \sin (x-a) .
\end{aligned}\right.
$$

where:

$$
A=\frac{\alpha L(\beta-1+\cos \beta \alpha L)-\sin \beta \alpha L}{\alpha L \cos \alpha L-\sin \alpha L}, B=\frac{\alpha L \sin \alpha L+\cos \alpha L-1}{\alpha L \cos \alpha L-\sin \alpha L} .
$$

For the estimation of the effectiveness of the method of control of the elastic-deformable state after the application of bending moments to the shaft face, and of the technological capabilities of such control, the relation describing the elastic line of the shaft was obtained in the form of (15).

The initial parameters are determined from the relation:

$$
\left\{\begin{array}{l}
y_{0}^{\prime}=\frac{F_{z g}}{\alpha F_{x 1}}\left(\beta-\frac{\sin \beta \alpha L}{\sin \alpha L}\right)-\frac{2 M_{2}}{F_{x 1}}\left(\frac{\cos \alpha L}{\sin \alpha L}-\frac{1}{\alpha L}\right), \\
y_{0}^{\prime \prime}=-\frac{2 M_{2}}{F_{x 1}}, \\
y_{0}^{\prime \prime \prime}=\frac{F_{z g}}{\alpha F_{x 1}}\left(\frac{\sin \beta \alpha L}{\sin \alpha L}\right)-\frac{2 M_{2}}{F_{x 1}}\left(\frac{\cos \alpha L}{\sin \alpha L}\right) .
\end{array}\right.
$$


Taking into account equation (19), the function of deformations ultimately assumes the form:

$$
\left\{\begin{aligned}
& y_{I}^{\prime}=\frac{F_{z g}}{\alpha F_{x 1}}(\beta-C) x-\frac{2 M_{2}}{F_{x 1}}\left(D-\frac{1}{\alpha L}\right) \alpha x-\frac{2 M_{2}}{F_{x 1}}(1-\cos \alpha)+ \\
&+\left(\frac{F_{z g} C}{\alpha F_{x 1}}+\frac{2 M_{2} D}{F_{x 1}}\right)(\alpha x-\sin \alpha x) \\
& y_{I I}(x)=y_{I}(x)-\frac{F_{z g}}{F_{x 1}}(x-a)+\frac{F_{z g}}{\alpha F_{x 1}} \sin (x-a)
\end{aligned}\right.
$$

where: $C=\frac{\sin \beta \alpha L}{\sin \alpha L}, D=\frac{\cos \alpha L}{\sin \alpha L}$.

Equations of lines of elastic deformations (20) and (22), as well as the equations presented in Table 1, describing the position and shape of a low-rigidity shaft in relation to its dimensions and active loads, permit estimation of the effectiveness of control of the elastic-deformable state of a low-rigidity shaft during turning.

The control of the elastic-deformable state with the use of the tensile force $F_{x 1}$ and eccentricity $e$ as the regulating factors, enabled the formulation of the problem of optimisation: definition of the values of the tensile force $F_{x 1}$ and eccentricity $e$ as functions of part parameters $L$ and $d$, component forces of machining $F_{f}, F_{p}, F_{c}$ (and thus also machining parameters $v, f, a_{p}$ ), distance $a$ from the cutting edge to the point of fixing of the part in the spindle and the instant coordinate $x$, minimising the deformation of the part:

$$
y=\min _{F x 1} \phi\left(d, e=F_{f}, v, a_{p}, f, F_{x 1}, e, a, x\right) .
$$

Function $y$ was arrived at on the basis of relations presented in Table 1 (columns 4 and 5). The values of components $F_{p}, F_{c}$ and $F_{f}$ of the machining force were defined by means of the technological conditions, i.e. the parameters and the geometry of machining; $u_{i}$ technological limitations imposed on the variable parameter $F_{x 1}$ resulting from the specific design features of the equipment and from the permissible tensile loads.

$$
\begin{gathered}
a \in\{0, L\}, \quad x \in\{0, L\}, \quad e \in\{0,2 d\} \\
F_{x 1} \in \bar{\sigma}=\left\{\left(F_{x 1}\right): g_{i}\left(F_{x 1}\right) \leq u_{i}\right\}, \quad u_{i} \geq 0, \quad i=1, n
\end{gathered}
$$

The most significant effect on the accuracy of machining is that of the value of deformation directly beneath the cutting tool; expressions (23) and (24) should be complemented with the limitation:

$$
a=L-x
$$


The formulated problem relates to issues of non-linear programming and can be solved with suitable methods [21]. The easiest way is to define the required values of the longitudinal tensile force $F_{x 1}$, at axial tension (model 2 or model 4 at $e=0$ ). For the selection of the values of $F_{x 1}$, corresponding to the relations (23), (24), (25), the bipartite method was applied. Numerical studies with the help of the method grids (Fig. 3a) demonstrated that the objective function $y$ is a unimodal function.

The bipartite method permits the determination of the extreme value of $F_{x 1}$ within:

$$
K_{i}=\log _{2}\left(F_{x 1 \max }-\frac{F_{x 1 \min }}{\Delta F_{x 1}}\right)
$$

steps or on the path of $(K-1)$ - fold calculation of the objective function $y$.

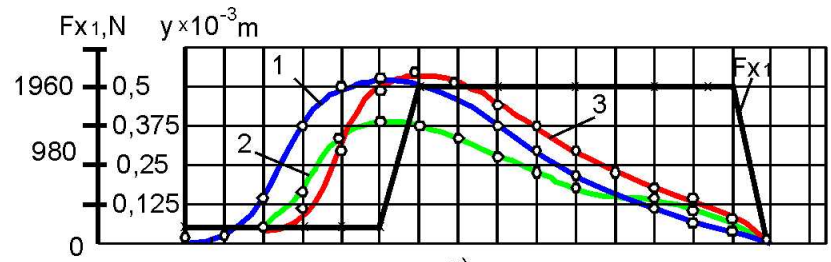

a)
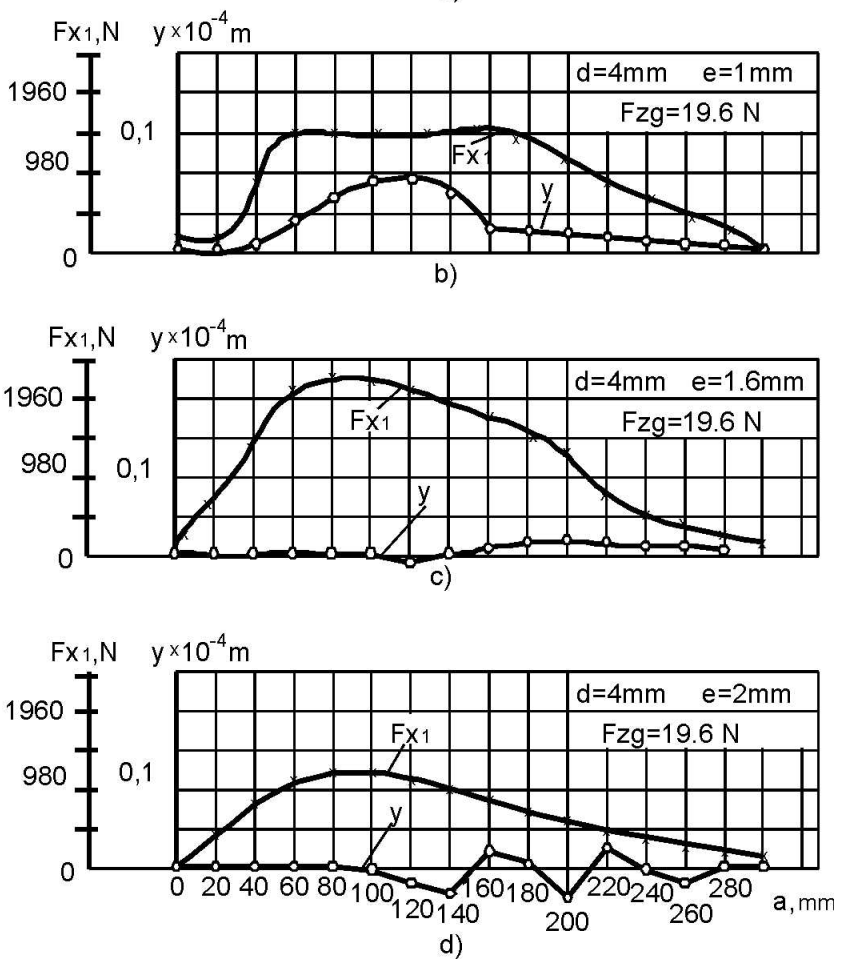

Figure 4 Objective functions and changes of tensile force $F_{x 1}$ in relation to the machining length obtained in the case of various models (1-3 - numbers designating models from Table 1). Note, different scale in Fig. 3a. 
The results of modelling (Fig. $3 \mathrm{~b}, \mathrm{c}, \mathrm{d}$ ) indicate the possibility of obtaining, determined by the process conditions, low values of elastic deformations as a result of controlling the force $F_{x 1}$, but with relation to the condition $(24)$ in such cases it is advisable to control $F_{x 1}$ at given values of eccentricity $e$ when testing model 4 (Martos, 1975).

To achieve the required low value of deformation the eccentric tension can be applied (model 4 at $e \neq 0)$.

Analysis of the results of numerical studies, obtained from relations (23), (24) and (25), as well as the supporting experimental tests, demonstrated that elastic deformations of low-rigidity shafts under eccentric tension control decrease, in the case of the parts class considered, from 2- to 20-fold, and in the case of shafts with $d<6 \mathrm{~mm}$ the decrease is achieved at lower values of the tensile force. It was demonstrated that eccentric tension very effectively reduces the elastic deformations of shafts with $d \geq 6 \mathrm{~mm}(L=300 \mathrm{~mm})$ as compared to axial tension. For example, at $d=8 \mathrm{~mm}$, $L=300 \mathrm{~mm}, F_{p}=147 \mathrm{~N}, \lambda_{1}=F_{p} / F_{f}=0.5\left(\kappa_{r}=90^{0}\right)$ and values of $F_{x 1}=980 \mathrm{~N}, e=2.5 \mathrm{~mm}$ (Fig. $4 \mathrm{~d})$ elastic deformations on the whole machining length are from 2- to 2.4-fold lower than in the case of axial tension. With adaptive control of the values of eccentricity $e$ and force $F_{x 1}$, the value of elastic deformations can be reduced 18-fold; it amounts to $(3-4.5) \cdot 10^{-2} \mathrm{~mm}(L=300 \mathrm{~mm}, d=8$ $\left.\mathrm{mm}, F_{p}=147 \mathrm{~N}, \lambda_{1}=0.5, F_{x 1}=1245 \mathrm{~N}, e=7.8 \mathrm{~mm}\right)$ and is practically stable over the whole length (Fig. 4e).

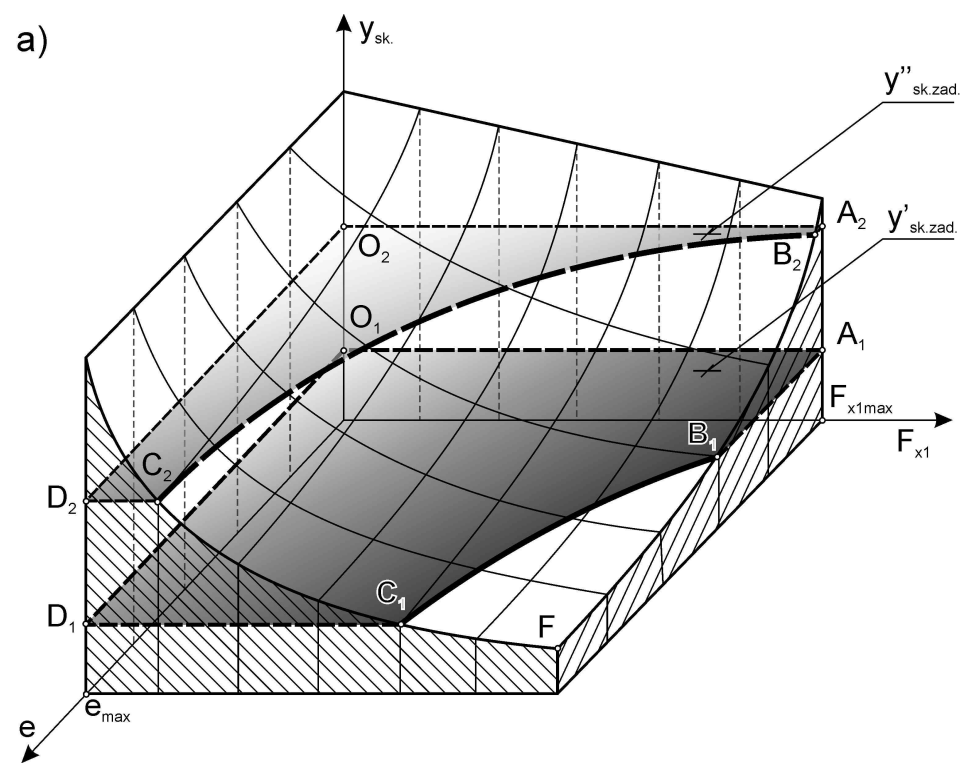

Figure 5 General view of 3D of hyperbololid like shape of the response surface for the values of the objective function - a); relations of change of objective function $y(0)$, tensile force $F_{x 1}(x)$ and eccentricity $e$ with relation to length $L$ at $x=a-b, c, d, e$ : b) $\left.\left.\left(d=6 \mathrm{~mm}, F_{z \mathrm{~g}}=49 \mathrm{~N}, F_{x 10}=980 \mathrm{~N}, L=300 \mathrm{~mm}, F_{f}=30 \mathrm{~N}\right), \mathrm{c}\right)\left(d=6 \mathrm{~mm}, F_{z g}=70 \mathrm{~N}, F_{x 10}=980 \mathrm{~N}, L=300 \mathrm{~mm}, F_{f}=40 \mathrm{~N}\right), \mathrm{d}\right)$ $\left(d=8 \mathrm{~mm}, F_{z g}=147 \mathrm{~N}, F_{x 10}=980 \mathrm{~N}, L=300 \mathrm{~mm}, F_{f}=196 \mathrm{~N}\right)$, e) $\left(d=8 \mathrm{~mm}, F_{z g}=147 \mathrm{~N}, F_{x 10}=980 \mathrm{~N}, L=300 \mathrm{~mm}, F_{f}=196 \mathrm{~N}\right)$. 


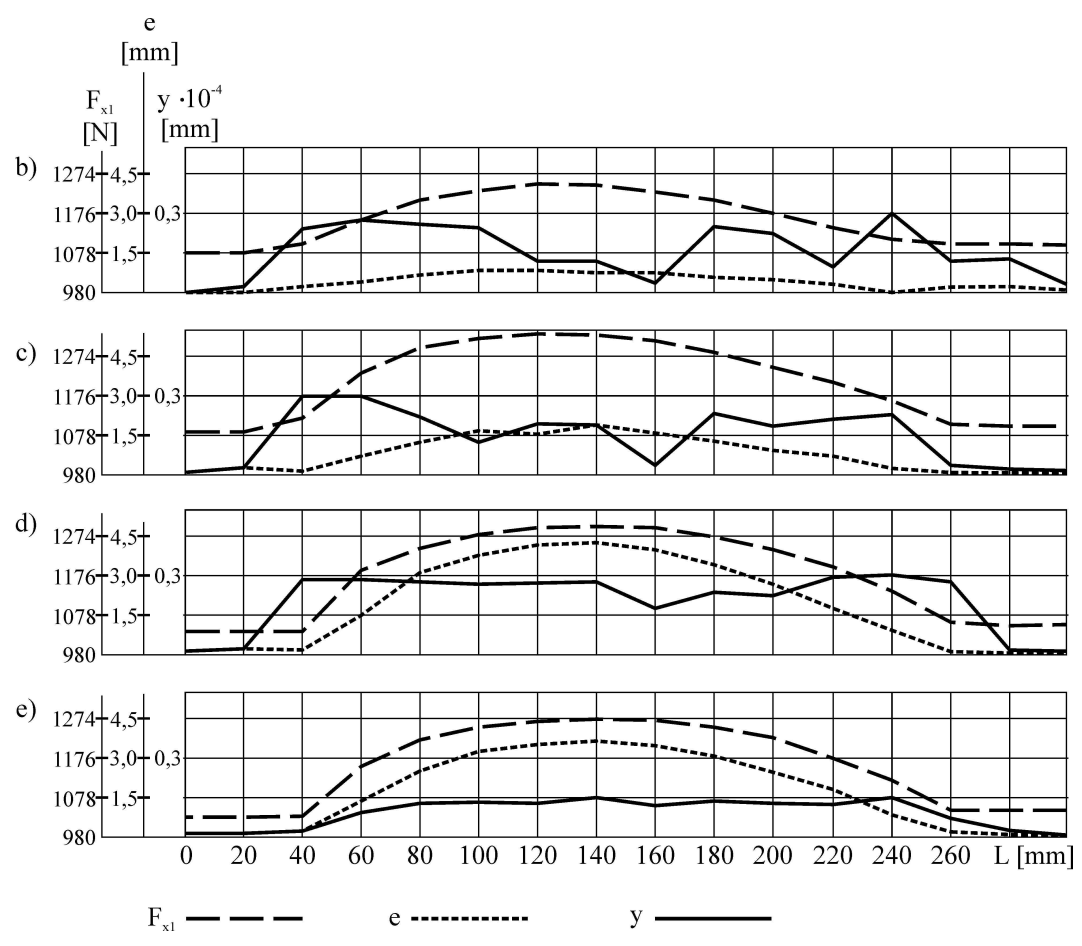

Figure 5 (continued)

To determine the optimum parameters $F_{x 1}$ and $e$, taking into account the limitations (24), (25), one can apply both the method of grids and (with relation to the unimodal nature of the objective function) the gradient method.

A general view of the numerically estimated 3D response surface for the objective function (23) with process limitations in the form of $F_{x 1 \max }, e_{\max }$, and with an extreme at point $F$ is presented in Fig. 4a. In many cases there is no need to find the extreme, and it is sufficient to determine the

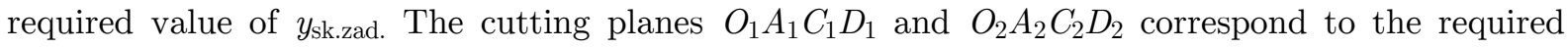
values of $y^{\prime}$ sk.zad. and $y^{\prime}$ 'sk.zad., relative to which the set of optimum values of $F_{x 1}$ and $e$ is sought.

The relations of changes of the objective function $y$, tensile force $F_{x 1}$ and eccentricity $e$ at various shaft diameters and values of machining force, obtained by means of modelling as a result of search with the gradients method, are presented in Fig. 4b,c,d,e. The modelling was conducted with the assumption of the following conditions: $d=6 \mathrm{~mm}, F_{z g}=49 \mathrm{~N}, F_{x 10}=980 \mathrm{~N}, F_{f}=30 \mathrm{~N}$ (Fig. $4 \mathrm{~b}$ ); $d=6 \mathrm{~mm}, F_{z g}=70 \mathrm{~N}, F_{x 10}=980 \mathrm{~N}, F_{f}=40 \mathrm{~N}$ (Fig. $4 \mathrm{c}$ ) $; d=8 \mathrm{~mm}, F_{z g}=147 \mathrm{~N}, F_{x 10}=980 \mathrm{~N}$, $F_{f}=196 \mathrm{~N}$ (Fig. $47 \mathrm{~d}$ ); $d=8 \mathrm{~mm}, F_{z g}=147 \mathrm{~N}, F_{x 10}=980 \mathrm{~N}, F_{f}=196 \mathrm{~N}$ (Fig. 4e).

As follows from analysis of the results presented in Fig 4, control of the level of machining accuracy, at elastic-deformable state of the part with $L / d=15-50$, can be effected with sufficient effectiveness through the control of two parameters: tensile force $F_{x 1}$ and eccentricity $e$; this permits the achievement of a theoretically assumed level of accuracy.

Loading a semi-finished product with a tensile force, causing the elastic-deformable state, is equivalent to the creation of an additional support causing an increase of the static stiffness of the part. Therefore, the alignment and the fixing of semi-finished products can be realized in selfcentring grips or in a spring sleeve. 


\section{CONCLUSIONS}

To determine the optimum parameters $F_{x 1}$ and $e$, taking into account the limitations (24), (25), one can apply both the method of grids and (with relation to the unimodal nature of the objective function) the gradient method.

A general view of the numerically estimated 3D response surface for the objective function (23) with process limitations in the form of $F_{x 1 \max }, e_{\max }$, and with an extreme at point $F$ is presented in Fig. 4a. In many cases there is no need to find the extreme, and it is sufficient to determine the

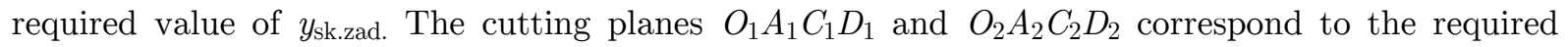

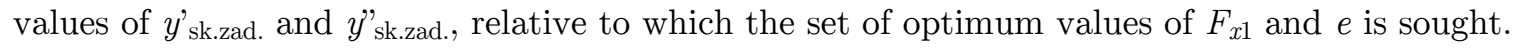

The relations of changes of the objective function $y$, tensile force $F_{x 1}$ and eccentricity $e$ at various shaft diameters and values of machining force, obtained by means of modelling as a result of search with the gradients method, are presented in Fig. 4b,c,d,e. The modelling was conducted with the assumption of the following conditions: $d=6 \mathrm{~mm}, F_{z g}=49 \mathrm{~N}, F_{x 10}=980 \mathrm{~N}, F_{f}=30 \mathrm{~N}$ (Fig. $4 \mathrm{~b}$ ); $d=6 \mathrm{~mm}, F_{z g}=70 \mathrm{~N}, F_{x 10}=980 \mathrm{~N}, F_{f}=40 \mathrm{~N}$ (Fig. $4 \mathrm{c}$ ) $; d=8 \mathrm{~mm}, F_{z g}=147 \mathrm{~N}, F_{x 10}=980 \mathrm{~N}$, $F_{f}=196 \mathrm{~N}$ (Fig. $47 \mathrm{~d}$ ); $d=8 \mathrm{~mm}, F_{z g}=147 \mathrm{~N}, F_{x 10}=980 \mathrm{~N}, F_{f}=196 \mathrm{~N}$ (Fig. 4e).

As follows from analysis of the results presented in Fig 4, control of the level of machining accuracy, at elastic-deformable state of the part with $L / d=15-50$, can be effected with sufficient effectiveness through the control of two parameters: tensile force $F_{x 1}$ and eccentricity $e$; this permits the achievement of a theoretically assumed level of accuracy.

Loading a semi-finished product with a tensile force, causing the elastic-deformable state, is equivalent to the creation of an additional support causing an increase of the static stiffness of the part. Therefore, the alignment and the fixing of semi-finished products can be realized in selfcentring grips or in a spring sleeve.

Acknowledement GL acknowledges the support by Structural Funds in the Operational Programme - Innovative Economy (IE OP) financed from the European Regional Development FundProject "Modern material technologies in aerospace industry", No. POIG.01.01.02-00-015/08-00.

\section{References}

Altintas Y (2000) Manufacturing automation: metal cutting mechanics, machine tool vibrations, and CNC design. Cambridge: Cambridge University Press.

Bisu CF, K'nevez JY, Darnis P, Laheurte R, Gerard A (2009) New method to characterize a machining system: application in turning. Int J Mat Form 2:93-105.

Bisu CF, Darnis P, Gerard A, K'nevez JY (2009) Displacements analysis of self-excited vibrations in turning. Int $J$ Advanc Manufac Tech 44:1-16.

Cahuc O, K'nevez JY, Gerard A, Darnis P, Albert G, Bisu CF, Gerard C (2010) Self-excited vibrations in turning: cutting moment analysis. Int $J$ Advanc Manufac Tech 47:217-225.

Cardi AA, Firpi HA, Bement MT, Liang SY (2008) Workpiece dynamic analysis and prediction during chatter of turning process. Mechanical Systems and Signal Processing 22: 1481-1494.

Chen CK, Tsao YM (2006) A stability analysis of regenerative chatter in turning process without using tailstock. Int J Advanc Manufac Tech 29:648-654. 
Halas W, Taranenko V, Swic A, Taranenko G (2008) Investigation of influence of grinding regimes on surface tension state. Lecture Notes In Artificial Intelligence, Vol. 5027, Berlin, Heidelberg: Springer - Verlag, pp. 749756 .

Han X, Ouyang H, Wang M, Hassan N, Mao Y (2012) Self-excited vibration of workpieces in a turning process. Proc IMech Part C: J Mech Engin Science 226:1958-1970.

Hassui A, Diniz AE (2003) Correlating surface roughness and vibration on plunge cylindrical grinding of steel, Int $J$ Mach Tools Manufac 43:855-862.

Jianliang G, Rongdi H (2006) A united model of diametral error in slender bar turning with a follower rest. Int $J$ Mach Tools Manufac 46:1002-1012.

Katz R, Lee CW, Ulsoy AG, Scott RA (1988) The dynamic response of a rotating shaft subject to a moving load, J Sound Vibr 122:131-148.

Li CJ, Ulsoy AG, Endres WJ (2003) The effect of flexible-tool rotation on regenerative instability in machining. $J$ Manuf Science Engineer 125:39-47.

Litak G, Rusinek R, Teter A (2004) Nonlinear analysis of experimental time series of a straight turning process. Meccanica 39:105-112.

Litak G, Rusinek R (2012) Dynamics of a stainless steel turning process by statistical and recurrence analyses. Meccanica 47:1517-1526.

Litak G, Kecik K, Rusinek R (2013) Cutting force response in milling of Inconel: Analysis by wavelet and Hilbert-Huang transforms. Latin American Journal of Solids and Structures 10:133-140.

Martos B (1975) Nonlinear programming. Theory and methods, North-Holland, Amsterdam.

Movahhedy M, Mosaddegh P (2006) Prediction of chatter in high speed milling including gyroscopic effects. Int $J$ Machine Tools Manufacture 46:996-1001.

Ouyang H, Wang M (2007) A dynamic model for a rotating beam subjected to axially moving forces, J Sound Vibr 308:674-682.

Pus VE, Pigert R, Sosonkin VL (1982), Avtomaticeskie stanocnye sistemy, Moscow Masinostroenie.

Qiang LZ (2000) Finite difference calculations of the deformations of multi-diameter workpieces during turning. $J$ Mat Process Technol 98:310-316.

Tlusty J (2000) Manufacturing processes and equipment. Upper Sadde River, NJ: Prentice Hall.

Sen AK, Litak G, Syta A, Rusinek R (2013) Intermittency and multiscale dynamics in milling of fiber reinforced composites. Meccanica 48:738-789.

Świć A, Taranenko V, Wołos D (2010) New method for machining of low-rigidity shafts. Adv Manufac Scien Technol 34:59-71.

Świć A, Taranenko W, Szabelski J (2011) Modelling dynamic systems of low-rigid shaft grinding. Maintenance and Reliability 13-24.

Xiong GL, Yi JM, Zeng C, Guo HK, Li LX (2003) Study of gyroscopic effect of the spindle on stability characteristics of the milling system. J Manuf Process Tech 138:379-384.

Young W, Budynas R, Roark RJ ( 2003) Roark's Formulas for Stress and Strain. McGraw-Hill, New York. 